\section{RESPIRATORY SYSTEM RESISTANCE AND BRONCHODILATOR RESPONSE IN SMALL CHILDREN WITH RECURRENT WHEEZING AND ASTHMA}

T. Stoeva, D. Christova, R. Mitrova, J. Uzunova

University Hospital Lozenetz, Sofia, Bulgaria

The interrupter technique is one of the few lung function tests that can be used for assessment of airway calibre in small children .With this technique, measurements of the resistance of the respiratory system (Rint) can be carried out quickly, with minimal cooperation of the child. The aim of the present study was to measure expiratory interrupter resistance ( Rint exp ) before and after bronchodilator administration in small children with recurrent wheezing or/and asthma. 40 children under 5 years of age were evaluated using Micro Medical devices according to producer requirements - Baseline Rint values were significantly greater in children with atopic background than in nonatopic children. Significant change in resistance following bronchodilator was found in 30 children and was greater in children with doctor diagnosis asthma than in those with recurrent wheezing only but not asthma. The measurement of airways resistance by the interrupter technique is clinically meaningful following an intervention such as the administration of bronchodilator.

\section{1}

\section{THE SEVERITY THRESHOLD OF CHILDHOOD ASTHMA - PEDIATRIC PULMONOLOGISTS' CRITERIA FOR ITS DEFINITION}

\author{
Z. Horev' ${ }^{1}$, B. Haim², H. Bibi²
}

${ }^{1}$ Director, Pediatrics in the Community, Division of Medicine, Clalit Health Services, Raanana, ${ }^{2}$ Pediatrics, Carmel Hospital, Haifa, Haifa, Israel

Asthma exacerbations in children may be treated in the ambulatory setting until a certain severity threshold is reached when ambulatory management is no longer safe.

Objective: To evaluate the criteria used by pediatric pulmonologists to define the severity threshold in the management of acute asthma.

Material \& methods: Registered Israeli pediatric pulmonologists filled an acute asthma oriented, decision making questionnaire. The questionnaire was divided into 3 sections: the primary clinic, the emergency room and in-hospital approach.

Results: Thirty pulmonologists responded to the questionnaire. The most important referral criteria to the ER were: central cyanosis, markedly reduced air entry to the lungs, grunting. Other important criteria were: severe tachypnea, clinical dyspnea, O2 saturation below $91 \%$, and repeat visit following failure to comply with treatment. Wheezing and auscultation findings were not indications for referral to the ER, nor were parental factors. The abovementioned criteria plus normal pCO2 levels in blood gases analysis were indicative for hospitalization after ER assessment.

The most important criteria indicative of sufficient improvement to allow discharge from hospital were: no IV therapy, resolution of dyspnea, retractions, and grunting, and $\mathrm{O} 2$ saturation at room air above $93 \%$. Most specialists didn't use PEFR as a guide for assessment.

Conclusions: Pediatric pulmonologists rely mainly on the degree of hypoxia and dyspnea demonstrated by the child when deciding whether a child has crossed the severity threshold, and should be referred to the ER, hospitalized or discharged in the course of a severe asthma episode.

\section{2}

\section{A RETROSPECTIVE CASE NOTE AUDIT OF ACUTE ASTHMA ADMISSIONS TO THE PAEDIATRIC DEPARTMENT, CRAIGAVON AREA HOSPITAL, NORTHERN IRELAND}

\author{
K.A. McConnell \\ Paediatrics, Craigavon Area Hospital, \\ Portadown, UK
}

Background and aims: Acute wheeze and asthma are common reasons for paediatric admissions to hospital in the UK. The British Thoracic Society (BTS) guidelines detail the preferred management of children admitted with acute wheeze/ asthma. National data has been collected yearly. Over one month (November 2009), this audit determined whether departmental practice followed BTS guidelines and sought to identify areas for improvement. Data collected to be compared with that from other UK hospitals participating in the national BTS asthma audit. 